\title{
Canada's Dominion Astrophysical Observatory and the rise of 20th Century Astrophysics and Technology
}

\author{
James E. Hesser, David Bohlender \& Dennis Crabtree \\ Dominion Astrophysical Observatory, Herzberg Astronomy and Astrophysics, \\ National Research Council of Canada, Victoria, BC, Canada, V9E 2E7 \\ email: jim.hesser@nrc-cnrc.gc.ca
}

\begin{abstract}
Construction of Canada's Dominion Astrophysical Observatory (DAO) commenced in 1914 with first light on 6 May 1918. As distinct from the contemporaneous development with private funding of major observatories in the western United States, DAO was (and remains) funded by the federal government. Canada's initial foray into 'big science', creation of DAO during the First World War was driven by Canada's desire to contribute significantly to the international rise of observational astrophysics enabled by photographic spectroscopy. In 2009 the Observatory was designated a National Historic Site. DAO's varied, rich contributions to the astronomical heritage of the 20th century continue in the 21st century, with particularly strong ties to Maunakea.
\end{abstract}

Keywords. General: history and philosophy of astronomy; Instrumentation: adaptive optics, spectrographs; Astronomical databases: miscellaneous

\section{Introduction}

Principles and practices behind DAO's high scientific and technological impact trace back to the founding Director, John Stanley Plaskett (1865-1941). He worked in close collaboration with the Warner and Swasey Company and the Brashear Optical Company to design and test the $1.82 \mathrm{~m}$ telescope today bearing his name. Indeed, perusal of Publications of the Dominion Astrophysical Observatory Volume 1 suggests that in modern parlance Plaskett acted as the "systems engineer," "project scientist" and "program manager" for the Observatory's development, commissioning and scientific operations until his 1935 retirement. The design was copied six times around the globe into the 1960s, reflecting high respect for the facility whose development Plaskett led.

\section{Scientific Programs}

To ensure that Canada's new observatory would have immediate, as well as lasting, scientific impact, Plaskett consulted widely regarding the critically important challenges and opportunities emerging in stellar astrophysics. In the first two decades or so of DAO operation the tiny staff embarked upon ambitious programs that today would be characterized as 'Key Projects'. The study of spectroscopic binaries became an Observatory hallmark and continued through the 1980s and 1990s not only for traditional binaries, but also for identification and characterization of newly-discovered X-ray sources. Those early efforts included amassing over 15 years radial velocities of $\mathrm{O}$ and $\mathrm{B}$ stars that, interpreted by Plaskett and J.A. Pearce through Oort's ideas of differential rotation, determined the most reliable estimate of the size and mass of the Milky Way available until radio astronomical techniques emerged in the 1950s. The first organic molecule, $\mathrm{CH}$, in interstellar 
space was discovered by DAO astronomer Andrew McKellar, who also deduced from interstellar CN observations the first, puzzling estimate of $\sim 3 \mathrm{~K}$ for the temperature of interstellar space that presaged the discovery of the cosmic microwave background radiation. In 1962 DAO's spectroscopic facilities were significantly augmented by the commissioning of the $1.2 \mathrm{~m}$ aperture telescope feeding its coudé spectrograph (subsequently named the McKellar Spectrograph), which E.H. Richardson's brilliant optical innovations made competitive with high-dispersion instruments of much larger telescopes.

\section{Institutional Evolution}

Throughout its history DAO has welcomed scientists from throughout Canada and around the world to use its facilities for their research. While there have been enormous changes to the operational environment since 1918, Plaskett's high standards and vision for scientific and technological excellence and relevance continue.

In 1970 Canada's Parliament charged the National Research Council (NRC) to "operate and administer any astronomical observatories established or maintained by the Government of Canada." All Federal astronomical facilities, including DAO, were brought into the NRC, which created the Herzberg Institute of Astrophysics to carry out the new mandate. In 1974 Canada undertook its first formal international agreement to build the CFHT, which was followed later by the JCMT, Gemini Observatory, ALMA, TMT and the development of the SKA, as well as leading roles in the development of space astronomy facilities funded by the Canadian Space Agency. Today DAO hosts the headquarters of the diverse NRC Herzberg organization that functions as a national astronomy laboratory supporting Canadian participation in those international facilities and their use by university astronomers and students. In addition to scientific research, NRC Herzberg activities comprise a) oversight of Canadian telescope allocation committees, international agreements, industrial partnerships, etc.; b) the Astronomy Technology Program (ground, space instrumentation); c) the Radio Astronomy Program; the Optical Astronomy Program (which operates the DAO telescopes; d) the Canadian Gemini Office; the Canadian Astronomy Data Centre (CADC); and e) public outreach (now in collaboration with community organizations).

As in Plaskett's day, ancillary instrumentation is critical to scientific breakthroughs. His pioneering efforts on spectrograph design continue through DAO's contributions to the CFHT and Gemini spectrographs under the leadership of David Crampton and the instrumentation team whose development he led (1980s-2000s). Their efforts included the introduction of multi-object capabilities on workhorse CFHT (MOS/SIS, 1992) and Gemini (GMOS 2001, 2002) spectrographs which enabled Canadian astronomers and their international partners to become leaders in observational cosmology beginning in the 1990s. In the same era the instrumentation team began its highly successful efforts on adaptive optics (AO), e.g., CFHT's 1988 experimental High Resolution Camera, that enabled the first detection of Virgo Cluster Cepheids used to determine the Hubble Constant; the MOS/SIS spectrograph (CFHT 1992); PUEO (CFHT, 1996); the ALTAIR AO camera for Gemini North (2002); and the Gemini Planet Imager 2013. These efforts have involved experts from Canadian universities and international partners.

'Data archiving' in Plaskett's day meant careful storage of the photographic plates on which spectra were taken prior to the 1980s. He likely would be astonished by the CADC (established in 1986) which today hosts and distributes data via the internet from diverse ground- and space-based telescopes to some 7,000 users worldwide. Among CADC's holdings are spectra selected from DAO's > 100K photographic spectral archive that Elizabeth Griffin, David Balam and David Bohlender are digitizing. Twentieth century digitized 
photographic spectra are being used in research from ozone in the Earth's atmosphere to the properties of eclipsing spectroscopic binaries such as $\epsilon$ Aurigae observed over its 27 year orbit since the 1950 s.

\section{Current Perspective}

Another important heritage dating to DAO's 1918 opening is sharing staff astronomical research with the public. Throughout its history the Observatory's outreach has functioned in synergy with members (predominantly amateur astronomers) of the Victoria Centre of the Royal Astronomical Society of Canada (RASC). In 2001 NRC opened a dedicated visitor facility, the Centre of the Universe (CU), to offer public outreach plus programming delivered by professional informal educators that was aligned with the school curriculum to complement and enrich the formal educational system. A change in NRC management philosophy led to CU closure in mid 2013. Today the Observatory is working with community organizations towards restoration of $\mathrm{CU}$ programming while individual staff continue to respond to frequent requests from community groups and schools to share their knowledge.

As prescient as Plaskett was, he could not have foreseen that a century later much of DAO's scientific, engineering and technical impact and heritage would have arisen from opportunities provided to international astronomy, including Canada, through access to Maunakea, for which Canadian astronomers are extremely grateful. Mahalo! 